\title{
Curvas de crescimento intra-uterino de uma população de alto nível socioeconômico
}

\author{
Intrauterine growth curves in a high-income population
}

\author{
Conceição A.M. Segre ${ }^{1}$, Gloria M.D.D. Colletto ${ }^{2}$, José R.D. Bertagnon ${ }^{3}$
}

\begin{abstract}
Resumo
Objetivo : as curvas de percentil constituem uma das formas de avaliação do crescimento intra-uterino e podem predizer doenças do recém-nascido como também caracterizar uma população. Este trabalho teve por objetivo construir as curvas de crescimento intrauterino dos recém-nascidos da Maternidade do Hospital Albert Einstein (MAE), hospital que atende a uma população de alto nível socioeconômico, e comparar com as curvas de crescimento intrauterino de uma população norte-americana da Califórnia.
\end{abstract}

Métodos: foram construídas curvas de crescimento intra-uterino a partir do peso do recém-nascido de parto único, tomado logo após o nascimento, e da idade gestacional segundo informações maternas, a partir da $32^{\mathrm{a}}$. semana de idade gestacional, abrangendo os nascimentos ocorridos na MAE no período de fevereiro de 1995 a fevereiro de 1999. Foram calculados os percentis 10, 50 e 90 do peso ao nascer para cada idade gestacional e comparados com os das curvas da Califórnia.

Resultados: as curvas dos percentis 10 e 50 na população da MAE não diferiram das curvas da Califórnia. Para o percentil 90, a curva da MAE ficou abaixo das curvas da Califórnia. Houve número menor de pequenos e grandes para a idade gestacional (PIG e GIG) quando classificados pelas curvas da Califórnia. A classificação em PIG, AIG, GIG mostrou-se relacionada significantemente com o ganho de peso materno nos dois sexos.

Conclusões: as duas populações analisadas segundo as curvas de crescimento intra-uterino são diferentes entre si; deverão ser identificados fatores específicos que atuem na população da MAE.

J Pediatr (Rio J) 2001; 77 (3): 169-74: recém-nascido, desenvolvimento infantil.

\section{Introdução}

$\mathrm{O}$ crescimento intra-uterino se caracteriza por crescimento e maturação orgânica e obedece a padrões reconhe-

\footnotetext{
1. Livre Docente em Pediatria Neonatal / EPM-UNIFESP. Coordenadora do curso de Pós-Graduação em Perinatologia do Inst. de Ensino e Pesquisa da Soc. Beneficente Israelita Brasileira Hosp. Albert Einstein.

2. Doutora em Ciências /USP.

3. Mestre em Saúde Pública / USP. Médico da Coordenação e Administração de Serviços em Saúde (COAS) da Pref. Municipal de São Paulo.
}

\begin{abstract}
Objective: growth curves can be used to assess intrauterine growth, to predict diseases in newborns, and to characterize different populations. The objective of this study was to obtain intrauterine growth curves of newborns from the Hospital Albert Einstein's Maternity Unit (MAE) and compare them with intrauterine growth curves of a population from California, USA.

Methods: we plotted the growth curves according to weight at birth and gestational age, which was obtained according to information from the mother, of newborns with over 32 weeks of term and from February 1995 to February 1999. We calculated the 10, 50, and $90^{\text {th }}$ percentiles of weight at birth for each gestational age and compared them with those of the growth curves from California.

Results: the growth curves for the $10^{\text {th }}$ and the $50^{\text {th }}$ percentiles did not differ from the growth curves from California. For the $90^{\text {th }}$ percentile, however, the MAE curves were lower than that from California. The MAE population presented less small-for-gestational age (SGA) and large-for-gestational age (LGA) babies when assessed according to the California curves . The categories of SGA, normal, and LGA for both male and female babies indicated a statistically significant relation with the weight gain of mothers.

Conclusions: the two populations assessed in this study were different according to intrauterine growth curves. Further studies should be carried out in order to identify specific factors that may concern the MAE population.
\end{abstract}

J Pediatr (Rio J) 2001; 77 (3): 169-74: newborn, child development.

cidos, sendo determinado individualmente pelo potencial genético e pela ação de fatores que regulam a utilização de nutrientes. Do equilíbrio dessas variáveis dependerá o peso ao nascer ${ }^{1}$. Atualmente, o acompanhamento do crescimento fetal tornou-se a principal questão na vigilância prénatal, não somente por traduzir a condição de bem-estar fetal, como por identificar a potente associação existente entre restrição do crescimento intra-uterino e natimortalidade $^{2,3}$. 
A tentativa de se identificar o mais precocemente possível as variações do crescimento fetal vem de longa data. Lubchenco e col. ${ }^{4}$, em 1963, apresentaram curvas de percentis de crescimento fetal, correlacionando morbidade e mortalidade com os padrões por eles estabelecidos. Em 1967, o Comitê de Feto e Recém-nascido da Academia Americana de Pediatria ${ }^{5}$ reconhece três parâmetros para a classificação do recém-nascido: peso, idade gestacional e crescimento intra-uterino. A partir daí, as curvas de crescimento se tornaram instrumentos indispensáveis e de grande valia em unidades neonatais. Inúmeros autores passaram a apresentar curvas de crescimento intra-uterino, tornandose clássicas as de Lubchenco e col. ${ }^{4}$, Gruenwald ${ }^{6}$, Usher e Mac Lean ${ }^{7}$, Babson e Benda ${ }^{8}$, Williams e col. ${ }^{9}$. Essa última, por ter sido construída com uma população de 2.288.806 recém-nascidos da Califórnia, permitiu uma determinação mais precisa da viabilidade fetal segundo diferentes combinações de peso e idade gestacional, sendo utilizada como padrão em vários serviços de neonatologia 9 .

Usando-se, portanto, a relação peso ao nascer/idade gestacional, o peso do recém-nascido pode ser categorizado como pequeno para a idade gestacional (se estiver abaixo do percentil 10), adequado (se estiver entre o percentil 10 e 90) e grande (se estiver acima do percentil 90) ${ }^{10}$. Dessa forma, os neonatologistas poderão identificar e antecipar prontamente problemas clínicos mais freqüentemente relacionados a uma determinada categoria, con-forme estejam associados a um padrão de crescimento intra-uterino.

A classificação de um recém-nascido segundo uma curva de crescimento deveria, preferencialmente, se reportar àquela construída com dados da população à qual pertence o indivíduo, pois, ao se classificar um determinado recém-nascido em um curva construída com população dissimilar, poder-se-ia estar incorrendo em grave erro ${ }^{11}$. Por outro lado, as curvas de percentis necessitam revisão periódica, em função de mudanças na miscigenação populacional e dos fatores socioeconômicos que atuam de forma temporal numa determinada população ${ }^{12}$.

O presente trabalho teve por objetivo construir as curvas de crescimento intra-uterino de uma população de alto nível socioeconômico na cidade de São Paulo e compará-las, para fins de caracterizar a população em estudo, com curvas internacionalmente conhecidas, quais sejam, as da Califórnia.

\section{Métodos}

Para a construção das curvas de percentis referentes à população de recém-nascidos dados à luz na Maternidade do Hospital Israelita Albert Einstein (MAE), realizou-se um estudo retrospectivo, onde foram incluídos os dados relativos ao peso obtido logo após o nascimento e à idade gestacional de todos os recém-nascidos vivos entre fevereiro de 1995 e fevereiro de 1999, entre 32 e 42 semanas de idade gestacional, totalizando 7.925 partos. Esta maternidade atende a uma população de gestantes caracterizada pelo atendimento pré-natal a $100 \%$ das mesmas, sendo que 94,6\% fizeram 6 consultas ou mais; $61,1 \%$ tinham curso superior completo; $97,9 \%$ eram possuidoras de seguro saúde; $0,9 \%$ eram adolescentes e a idade média das mães foi de 31,1 anos; 68,2\% não eram fumantes. Esses dados foram obtidos a partir das informações coletadas de um banco de dados da Unidade Neonatal, digitados em um programa EPI-Info 6.0. Foram excluídos os recém-nascidos com menos de 32 semanas de idade gestacional, em função do pequeno número de casos, os recém-nascidos com malformações congênitas maiores e os gemelares.

O recém-nascido foi pesado logo após o nascimento por funcionária de enfermagem, em balança da marca SEMCO $^{\circledR}$, elétrica, digital com capacidade de $10 \mathrm{~kg}$ e sensibilidade de $5 \mathrm{~g}$, e seu valor imediatamente anotado no prontuário perinatal então vigente na Unidade Neonatal (história clínica perinatal - HCP). A idade gestacional foi obtida a partir das informações maternas sobre a data do primeiro dia da última menstruação normal e calculada segundo a regra de Naegele, transformando-se o número de dias em semanas completas.

Todos os dados utilizados neste trabalho, incluindo o peso inicial e final das gestantes, foram coletados do banco de dados da Unidade Neonatal, alimentado a partir das anotações feitas na História Clínica Perinatal (HCP) e digitados em um programa EPI-Info 6.0 por um dos médicos da equipe.

Para fins de comparação foram utilizadas as curvas da Califórnia, selecionadas para tal por terem sido construídas a partir dos dados de peso e idade gestacional de uma população branca em sua maioria, e abrangendo 2.288.806 crianças.

\section{Metodologia estatística}

Os dados foram analisados utilizando-se o programa estatístico SPSS e o Curve Expert 1.3. Foram calculados os percentis 10,50 e 90 do peso ao nascer, para cada idade gestacional. A seguir foram feitos os ajustes dessas curvas, por meio da regressão quadrática, para eliminar pequenas distorções, encontradas principalmente nas idades gestacionais mais baixas ( 32 a 35 semanas), devido ao pequeno número de casos nesse grupo.

Este estudo foi aprovado pelo Comitê de Ética em Pesquisa da Instituição e, por se tratar de análise de dados a partir de prontuários, não necessitou de consentimento informado dos pacientes.

\section{Resultados}

Foram incluídos no estudo 7.925 recém-nascidos, sendo 3.972 do sexo masculino e 3.953 do sexo feminino. A Tabela 1 mostra as médias de peso dos RN (masculino e feminino) classificados por semanas de idade gestacional. 
O peso médio dos RN masculinos foi de 3.305,54 $\pm 448,76$ e dos femininos foi de $3.180,86 \pm 444,46$. As Figuras $1 \mathrm{~A} \mathrm{e}$ $1 \mathrm{~B}$ mostram as curvas dos percentis 10, 50 e 90 da MAE para os sexos masculino e feminino respectivamente. As Figuras 2A e 2B mostram a comparação gráfica entre as curvas dos percentis 10,50 e 90 para a MAE e os dados das curvas da Califórnia para os sexos masculino e feminino.

Tabela 1 - Médias (X) e desvios-padrão (D.P.) dos pesos dos $\mathrm{RN}$ para o sexo masculino e feminino

\begin{tabular}{lrcrc}
\hline & \multicolumn{2}{c}{ Masculino } & \multicolumn{2}{c}{ Feminino } \\
Semanas & N & X \pm D.P. & N & X \pm D.P. \\
\hline 32 & 12 & $1826,7 \pm 440,0$ & 9 & $1826,7 \pm 418,6$ \\
$\mathbf{3 3}$ & 16 & $1955,2 \pm 405,9$ & 21 & $1955,2 \pm 376,3$ \\
$\mathbf{3 4}$ & 24 & $2130,5 \pm 457,1$ & 29 & $2130,5 \pm 463,5$ \\
$\mathbf{3 5}$ & 43 & $2560,7 \pm 495,2$ & 43 & $2560,7 \pm 466,5$ \\
$\mathbf{3 6}$ & 125 & $2635,3 \pm 379,8$ & 117 & $2635,3 \pm 427,3$ \\
$\mathbf{3 7}$ & 301 & $2899,6 \pm 396,4$ & 277 & $2899,6 \pm 404,0$ \\
$\mathbf{3 8}$ & 875 & $3109,4 \pm 393,2$ & 844 & $3109,5 \pm 388,9$ \\
$\mathbf{3 9}$ & 1.485 & $3245,3 \pm 381,4$ & 1.458 & $3245,3 \pm 374,3$ \\
$\mathbf{4 0}$ & 922 & $3344,0 \pm 393,7$ & 957 & $3344,1 \pm 373,7$ \\
$\mathbf{4 1}$ & 152 & $3415,1 \pm 401,2$ & 179 & $3415,1 \pm 387,0$ \\
$\mathbf{4 2}$ & 17 & $3446,8 \pm 484,4$ & 19 & $3446,8 \pm 402,7$ \\
\hline Total & 3.972 & $3180,9 \pm 448,8$ & 3.953 & $3180,9 \pm 444,5$ \\
\hline
\end{tabular}

Na Tabela 2 acham-se os dados relativos à classificação dos RN da MAE em pequenos, adequados ou grandes para a idade gestacional (PIG, AIG, GIG) segundo as curvas da Califórnia e de acordo com os sexos. Pode-se observar que existe uma diminuição das frequiências das classes PIG e GIG em relação ao esperado (10\%) em comparação com as curvas da Califórnia.

As médias de ganho de peso materno (GPM) durante a gestação nos três grupos (PIG, AIG e GIG) se encontram na Tabela 3. A comparação dessas médias por análise de variância mostra que elas são significantemente diferentes entre si, para crianças tanto do sexo masculino quanto do sexo feminino ( $F=60,5$ e 50,6 respectivamente; $p<0,001$ para ambos os sexos).

A Tabela 4 mostra a distribuição das percentagens de recém-nascidos PIG e AIG/GIG (analisados agora conjuntamente, em função do pequeno número de recém-nascidos GIG), classificados segundo os próprios dados da MAE, de acordo com a faixa de ganho de peso materno. Pode-se observar que, dentro de cada classe, as maiores percentagens de PIG estão nas faixas de menor ganho de peso materno. Corroborando esses achados, observa-se que existe uma correlação positiva entre o peso do recémnascido e o ganho de peso materno durante a gestação $\left(\mathrm{r}_{\text {Spearman }}=0,219 ; \mathrm{p}<0,001\right)$.
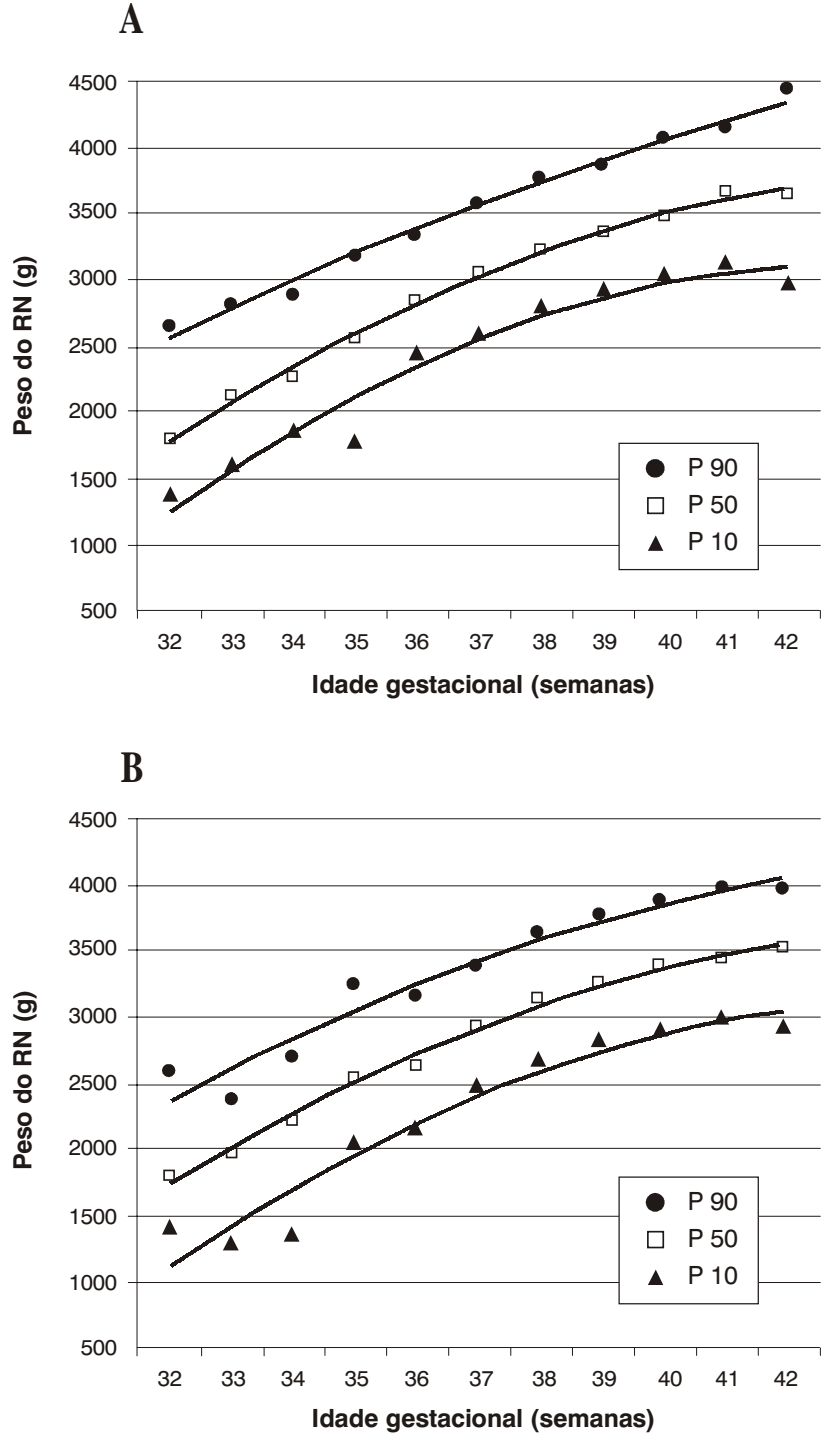

Figura 1 - Curvas de distribuição dos valores observados e ajustados dos percentis 10,50 e 90 para o sexo masculino (A) e feminino (B) dos recém-nascidos da Maternidade Albert Einstein

O melhor modelo para a estimativa do ganho de peso do $\mathrm{RN}$ em relação ao ganho de peso materno é a regressão cúbica: $\mathrm{Y}=2.846,78+35,9654 \times \mathrm{GPM}-0,2295 \times \mathrm{GPM}^{2}$ $\left.+0,0099 \times \mathrm{GPM}^{3}, \mathrm{~F}=131,03 ; \mathrm{p}<0,001\right)$. A Tabela 3 apresenta os valores estimados por esta regressão para o peso do RN.

A Figura 3 representa os valores teóricos da regressão cúbica, mostrando o peso do recém-nascido em relação ao GPM. Pode-se observar que ocorre aumento de peso do recém-nascido até o valor de $28 \mathrm{~kg}$ de GPM e que, a partir daí, o peso do recém-nascido começa a decrescer. No entanto, no intervalo de 16 a $28 \mathrm{~kg}$, o aumento de peso do recém-nascido é de apenas 153,4g. 
A

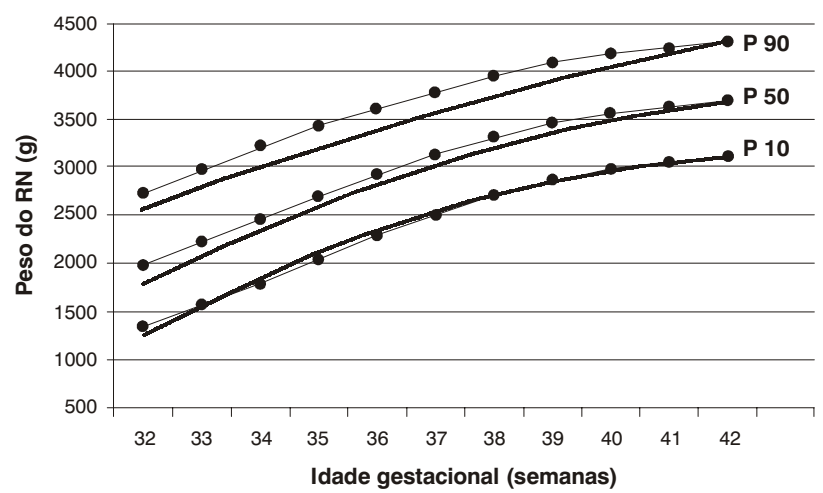

B

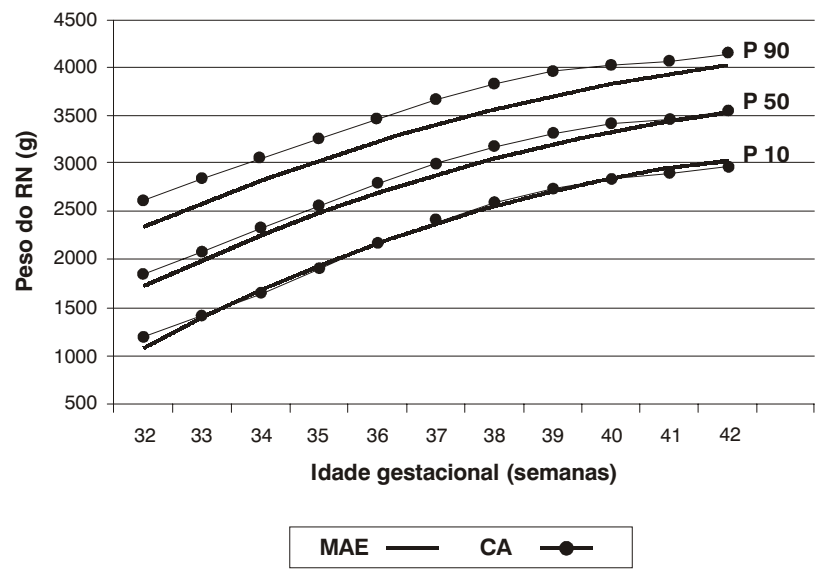

Figura 2 - Curvas de distribuição dos percentis 10, 50 e 90 da Maternidade Albert Einstein (MAE) e da Califórnia (CA) para o sexo masculino (A) e feminino (B)

\section{Discussão}

A relação entre a idade gestacional e o peso de um recém-nascido reflete a adequação do crescimento intrauterino, facilitando ao neonatologista a antecipação de problemas clínicos e mesmo da mortalidade neonatal, permitindo que medidas, não somente curativas, mas também preventivas possam ser tomadas em tempo hábil. Por outro lado, o crescimento intra-uterino traduz, ainda, caracterís-

Tabela 2 - Classificação dos RN da MAE segundo a curva da Califórnia, para os dois sexos

\begin{tabular}{lcrcr}
\hline & \multicolumn{2}{c}{ Sexo Masculino } & \multicolumn{2}{c}{ Sexo Feminino } \\
& Freqüência & \% & Freqüência & \multicolumn{1}{c}{$\%$} \\
\hline PIG & 268 & 6,7 & 290 & 7,3 \\
AIG & 3.526 & 88,8 & 3.505 & 88,7 \\
GIG & 178 & 4,5 & 158 & 4,0 \\
\hline Total & 3.972 & 100,0 & 3.953 & 100,0 \\
\hline
\end{tabular}

Tabela 3 - Aumento médio de peso das gestantes, de acordo com a classificação do RN, classificados pelos dados da própria MAE - figura 1

\begin{tabular}{lrrrrrr}
\hline & \multicolumn{3}{c}{ Sexo masculino } & \multicolumn{3}{c}{ Sexo feminino } \\
& \multicolumn{1}{c}{ N } & Média & D.P. & \multicolumn{1}{c}{ N } & Média & D.P. \\
\hline PIG & 397 & 11,99 & 3,83 & 398 & 11,83 & 4,26 \\
AIG & 3.161 & 13,09 & 4,26 & 3.158 & 12,89 & 4,31 \\
GIG & 389 & 15,24 & 5,06 & 381 & 14,88 & 4,96 \\
\hline Total & 3.947 & 13,19 & 4,37 & 3.937 & 12,98 & 4,43 \\
\hline
\end{tabular}

ticas populacionais que, por sua vez, se acham relacionadas a questões nutricionais, ambientais, de hábitos e estilo de vida de uma população ${ }^{10}$. O Comitê de Feto e RecémNascido da Academia Americana de Pediatria ${ }^{13}$, em 1988, recomendou que todos os recém-nascidos fossem classificados, levando-se em consideração seu crescimento intrauterino.

A construção de curvas de crescimento intra-uterino relativas a uma determinada população poderá revelar suas características específicas, evitando possíveis erros decorrentes da classificação de um recém-nascido segundo uma curva que não é adequada à população a qual pertence esta criança em particular ${ }^{11}$. Contudo, é preciso que se tenha em mente que o crescimento intra-uterino deriva da somatória de fatores genéticos e ambientais, e que estes últimos poderão atuar de tal forma a mascarar o potencial genético de um determinado indivíduo ${ }^{14}$, Beiguelman e col. ${ }^{15}$, em 1998, comparando os dados de três hospitais de diferentes classes sociais, sugerem que o efeito genético fetal sobre o peso ao nascer teria maior oportunidade de se manifestar nas mães subnutridas, pois o coeficiente de herdabilidade estimado foi de 0,52 para o hospital de menor nível socioeconômico, enquanto os outros dois não mostraram efeito genético no peso ao nascer.

Embora haja muitas controvérsias em relação a uma classificação satisfatória de classe social, a população de gestantes incluída no presente estudo foi considerada como pertencente a uma classe social elevada, segundo análise de seu grau de educação, conforme se acha referido na literatura $^{16-18}$, entendendo-se ainda como fatores que podem contribuir como indicativos de classe social elevada sua freqüência e número de consultas ao pré-natal. Assim, as curvas de crescimento intra-uterino referentes à população deste estudo foram comparadas às curvas da Califórnia, tomadas como padrão para comparações, não só pelo grande número de indivíduos envolvidos em sua construção, como também pelo fato de se referir a uma população de maioria branca $(85,6 \%)$, de origem espanhola ou não, com características razoavelmente homogêneas. As curvas de crescimento intra-uterino da MAE mostraram aspectos interessantes e peculiares em relação às curvas da Califór- 
Tabela 4 - Distribuição dos RN pequenos para a idade gestacional e adequados ou grandes, de acordo com a faixa de aumento de peso materno durante a gestação, e aumento de peso estimado para o RN

\begin{tabular}{|c|c|c|c|c|c|c|}
\hline \multicolumn{2}{|c|}{$\begin{array}{l}\text { Aumento de } \\
\text { peso materno }\end{array}$} & \multicolumn{2}{|c|}{ PIG } & \multicolumn{2}{|c|}{ AIG/GIG } & \multirow{2}{*}{$\begin{array}{l}\text { Aumento de } \\
\text { peso do RN } \\
\text { em g }\end{array}$} \\
\hline em kg & em \% & $\mathbf{N}$ & $\%$ & $\mathbf{N}$ & $\%$ & \\
\hline 1- 5 & 2,0 & 25 & 16,3 & 128 & 83,7 & 137,1 \\
\hline 6-10 & 26,8 & 288 & 13,6 & 1827 & 86,4 & 153,8 \\
\hline $11-15$ & 46,9 & 359 & 9,7 & 3341 & 90,3 & 127,4 \\
\hline $16-20$ & 18,8 & 100 & 6,7 & 1383 & 93,3 & 93,4 \\
\hline $21-25$ & 4,2 & 17 & 5,2 & 311 & 94,7 & 51,9 \\
\hline 26-30 & 1,0 & 6 & 7,8 & 71 & 92,2 & 2,9 \\
\hline $31-35$ & 0,3 & 0 & - & 24 & 100,0 & $-53,5$ \\
\hline $35-40$ & 0,0003 & 0 & - & 3 & 100,0 & $-117,5$ \\
\hline
\end{tabular}

nia. Pode-se observar que, para o percentil 10 , as duas curvas praticamente não diferiram, para ambos os sexos. As curvas do percentil 50 da Califórnia acham-se um pouco acima das curvas da MAE, até a $40^{\mathrm{a}}$ semana, não diferindo a partir daí. A maior diferença encontra-se, porém, em relação ao percentil 90, onde as curvas da MAE estão abaixo das curvas da Califórnia, embora para o sexo masculino haja também uma tendência de sobreposição nas semanas $41^{\mathrm{a}}$ e $42^{\mathrm{a}}$. A população da MAE é composta por gestantes que, em sua totalidade, fazem controle pré-natal. Neste controle existe a preocupação de se evitar um excesso de ganho ponderal, o que se evidencia pela média de ganho de peso durante a gestação, que foi de $13,1 \mathrm{~kg}$, o que é considerado como ganho moderado ${ }^{19}$. Este fato poderia explicar as diferenças encontradas no percentil $90 \mathrm{em}$ relação às curvas da Califórnia, podendo-se mesmo especular se este resultado não seria a tradução de uma restrição calórica "iatrogênica" sobre o peso fetal. Gregory e Rush ${ }^{20}$ não puderam relacionar a recomendação médica para ingestão de $1.800 \mathrm{kcal} / \mathrm{dia}$ ou menos, à restrição de crescimento intra-uterino. Contudo, pelo fato de estas gestantes ganharem mais peso que aquelas em dieta livre, concluíram que os resultados eram compatíveis com a não adesão das gestantes à dieta. Já em 1975, Simpson e col. ${ }^{21}$ relacionavam o ganho de peso durante a gestação ao peso do recémnascido de termo. Os achados destes autores coincidem com os do presente estudo, mostrando que existe realmente uma correlação entre GPM e peso do neonato. Gormican e $\mathrm{col}^{22}$, estudando os efeitos de ingestões calóricas restritas ou não, sobre o peso de nascimento, concluíram que a restrição calórica durante a gestação pode ser não somente indesejável como também deletéria para o recém-nascido. No estudo de Godfrey e col. ${ }^{23}$, gestantes que tiveram ingestão elevada de carboidratos no começo da gestação e baixa de proteínas derivadas do leite no final da gestação, deram à luz recém-nascidos mais magros. As associações encontradas por estes autores poderiam refletir restrições no desenvolvimento placentário impostas pela nutrição materna durante a gestação. Por outro lado, em estudo anterior, nesta mesma população atendida na MAE, foi demonstrado que um ganho de peso, durante a gestação, superior a $16 \mathrm{~kg}$ não aumentava o peso do recém-nascido além de um certo limite ${ }^{24}$. No presente estudo, com maior número de casos, verificou-se um aumento de peso dos recém-nascidos correspondente a um ganho de peso materno de até $27 \mathrm{~kg}$; contudo, a partir de $16 \mathrm{~kg}$, este ganho foi mínimo (153g) e teria sido detectado em função do maior número de casos, além do fato de que os gêmeos foram excluídos no atual estudo. Beiguelman e col. ${ }^{25}$, em 1998, demonstraram que, comparando-se o crescimento fetal de recém-nascidos de parto único e gemelar de três hospitais

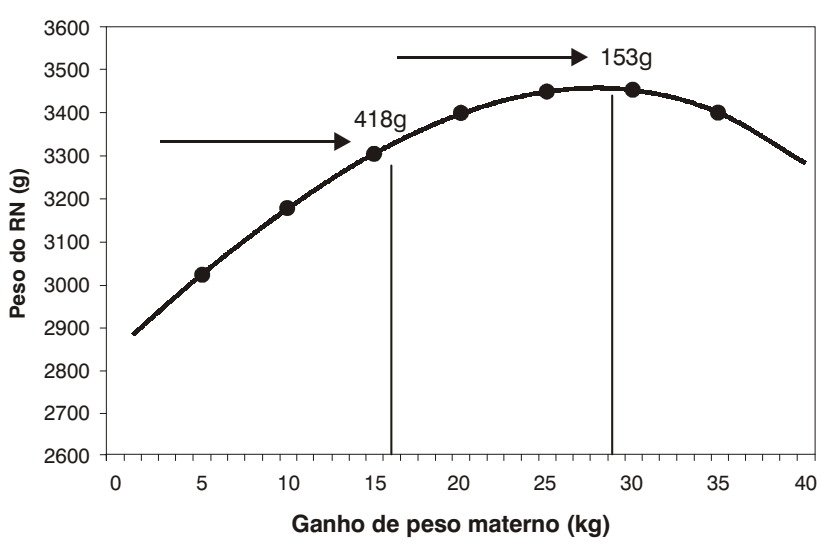

Figura 3 - Peso do recém-nascido estimado pela regressão cúbica, em função do ganho de peso materno. Entre 1 e $15 \mathrm{~kg}$ de ganho de peso materno, o aumento estimado do peso do RN é de $418 \mathrm{~g}$, e de 16 a 28 kg é de $153 \mathrm{~g}$ 
de diferentes níveis socioeconômicos da cidade de São Paulo, o padrão da taxa de crescimento fetal dos gêmeos é retardado em relação aos recém-nascidos de parto único, independentemente do nível socioeconômico. No entanto, a idade gestacional em que começa esse atraso parece estar relacionada ao nível socioeconômico das mães e, por consequiência, com seu padrão alimentar, pois no nível socioeconômico mais baixo a diferença começa em idade gestacional menor (22 semanas), enquanto que, no de maior nível, o atraso começa em torno de 30 semanas.

Embora, segundo Jonhson e Yancey ${ }^{26}$, não haja efetivamente evidências de que um aumento de peso gestacional isoladamente possa melhorar o resultado perinatal, há que se salientar que esta afirmação não é de consenso na literatura, pois o aumento do peso não ocorre, na verdade, "isoladamente", mas é o resultado do consumo regular de alimentos diversos, que promovem um aporte nutricional completo e otimizam o desenvolvimento e crescimento fetal, indicados pelo peso de nascimento, mas não limitados exclusivamente àquele resultado ${ }^{27}$.

Classificando-se os recém-nascidos da MAE segundo as curvas da Califórnia, pode-se observar, também, menor número de RN PIG e GIG que na população de Williams e col. ${ }^{9}$ No caso da MAE, por tratar-se de uma população de alto nível socioeconômico, de pacientes com controle prénatal presumivelmente adequado, o número de recémnascidos PIG seria menor em função das boas condições maternas desde a fase pré-concepcional, bem como durante a gestação, de modo a contemplar condições gestacionais teoricamente ideais, que teriam reflexos nos resultados perinatais. O menor número de GIG observado na MAE poderia ser atribuído ao que foi anteriormente exposto sobre a restrição calórica imposta às gestantes.

Conclui-se, por este estudo, que a população da MAE é diferente da população do estudo de Williams e col. ${ }^{9}$ As diferenças encontradas evidenciam que fatores específicos podem estar atuando na população da MAE e devem merecer investigações subseqüentes.

\section{Referências bibliográficas}

1. Hay WW Jr, Catz CS, Grave GD, Yaffe SJ. Workshop summary: fetal growth: its regulation and disorders. Pediatrics 1997;99: 585-91.

2. Sparks JW, Ross JC, Cetin I. Intrauterine growth and nutrition. In: Polin RA, Fox WW, eds. Fetal and neonatal physiology. 2nd ed. Philadelphia: W.B. Saunders; 1998. p. 267-83.

3. Gardosi J. Customized growth curves. Clin Obstet Gynecol 1997;40:715-22.

4. Lubchenco LO, Hansman C, Dressler M, Boyed E. Intrauterine growth as estimated from live born birth weight data at 24-42 weeks gestation. Pediatrics 1963;32:793-9.

5. American Academy of Pediatrics, Committee on Fetus and Newborn. Nomenclature for duration of gestation, birth weight and intra-uterine growth. Pediatrics 1967;39:935-8.

6. Gruenwald P. Growth of the human fetus. Am J Obstet Gynecol 1966;94:1112- 9.
7. Usher R, McLean F. Intrauterine growth of live-born caucasian infants at sea level: standards obtained from measurements in 7 dimensions if infants born between 25 and 44 weeks of gestation. J Pediatr 1969;74:901-10.

8. Babson SG, Benda GI. Growth graphs for the clinical assessment of infants of varying gestational age. J Pediatr 1976;89:814-20.

9. Williams RL, Creasy RK, Cunningham GC, Hawes WE, Norris $\mathrm{FD}$, Tashiro M. Fetal growth and perinatal viability in California. Obstet Gynecol 1982;59:624-32.

10. Pittard WB. Classification of the low-birth-weight infant. In: Klaus MH, Fanaroff AA, eds. Care of the high risk neonate. 4th ed. Philadelphia: WB Saunders; 1993. p. 86-113.

11. David RJ. Population-based intrauterine growth curves from computerized birth certificates. South Med J 1983;76:1401-6.

12. Guaran RL, Wein P, Sheedy M, Walstab J, Beischer NA. Update growth percentiles for infants born in an Australian population. Aust N Z Obstet Gynaecol 1994;34:39-50.

13. American Academy of Pediatrics, Committee on Fetus and Newborn, and the American College of Obstetricians and Gynecologists, Committee on Obstetrics. Maternal Fetal Medicine: Guidelines for Perinatal care. Elk Gove Village, IL: American Academy of Pediatrics; 1988. p. 86.

14. Anderson MS, Hay WW Jr. Intrauterine growth restriction and the small-for-gestational-age infant. In: Avery GB, Fletcher MA, MacDonald MG, eds. Neonatology. Patophysiology and management of the newborn. 5th ed. Philadelphia: Lippincott Williams and Wilkins; 1999. p. 411-44.

15. Beiguelman B, Colletto GMDD, Franchi-Pinto C, Krieger H. Birth weight of twins: 2. Fetal genetic effect on birth weight. Genet Mol Biol 1998;21:155-8.

16. Zurayk H, Halabi S, Deeb M. Measuress of social class based on education for use in health studies in developing countries. J Epidemiol Community Health 1987;42:173-9.

17. Memminki E, Malin M, Rahkonen. Mother's social class and perinatal problems in a low-problem area. Int $\mathrm{J}$ Epidemiol 1990;19:983-90.

18. Alonso J, Perez P, Saez M, Murillo C. Validity of occupation as an indicator of social class according to the British Registar General Classification. Gac Sanit 1997;11:205-13.

19. Thorsdottir I, Birgisdottir BE. Different weight gain in women of normal weight before pregnancy: postpartum weight and birth weight. Obstet Gynecol 1998;92:377-83.

20. Gregory PB, Rush D. Iatrogenic caloric restriction in pregnancy and birthweight. Am J Perinat 1987;4:365-71.

21. Simpson JW, Lawless RW, Mitchel AC. Responsibility of the obstetrician to the fetus. II. Influence of prepregnancy weight and pregnancy weight gain on birth weight. Obstet Gynecol 1975;45:481-7.

22. Gormican A, Valentine J, Satter E. Relationships of maternal weight gain, prepregnancy weight, and infant birth weight. Interaction of weight factors in pregnancy. J Am Diet Assoc 1980;77:662-7.

23. Godfrey KM, Barker DJ, Robinson S, Osmond C. Maternal birthweight and diet in pregnancy in relation to the infant's thinness at birth. Br J Obstet Gynaecol 1997;104:663-7.

24. Lizo CLP, Azevedo-Lizo Z, Aronson E, Segre CAM. Relação entre ganho de peso materno e peso do recém-nascido. J Pediatr (Rio J) 1998;74:114-8.

25. Beiguelman B, Colletto GMDD, Franchi-Pinto C, Krieger H. Birth weight of twins: 1 . Fetal growth pattern of twins and singletons. Genet Mol Biol 1998;21:151-4.

26. Jonhson JWC, Yancey MK. A critique of the new recommendations for weight gain in pregnancy. Am J Obstet Gynecol 1996;174:254-8.

27. Lederman SA. Pregnancy weight gain not excessive. Letter. Am J Obstet Gynecol 1996;175:1395-6. 